\title{
Antimicrobial Resistant Streptococcus pneumoniae Infections: Clinical and Epidemiological Trends
}

\author{
James D. Kellner ${ }^{* \dagger}$, M.D., M.Sc., F.R.C.P.(C), and Donald E. Low ${ }^{\S}$ M.D., \\ F.R.C.P.(C)
}

\section{INTRODUCTION}

Infectious diseases remain an important cause of human morbidity and mortality. In the developing world, infections cause $43 \%$ of all deaths (1). In the developed world, only around $1 \%$ of deaths are caused by infections (1). However, deaths from infections increased by $58 \%$ in the United States from 1980 to 1992 (2). This increase has been largely due not only to human immunodeficiency virus (HIV) infections, but also to respiratory tract infections and to septicemia.

Complicating the persistent issue of infectious diseases is the increasing problem of resistance by bacteria, viruses and fungi to antimicrobial drugs. The fundamental factor that has lead to antimicrobial resistance is the widespread use of antimicrobials and the resultant selective pressure to promote evolution and transmission of resistant organisms. Increasing rates of resistance to common and important community and hospital acquired pathogens is occurring in Canada and worldwide (3). The impact of bacterial resistance to antimicrobial agents has not been clearly determined (4), although it appears that morbidity and mortality may be increased (3). The potential public health impact of antimicrobial resistance is enormous. While some antimicrobial resistant pathogens are only a threat to specific populations, e.g., Burkholderia cepacia infections in cystic fibrosis, others are common and potentially serious pathogens for the entire population $(5,6)$. The

\footnotetext{
* To whom correspondence should be addressed: Division of Infectious Diseases, Alberta Children's Hospital, 1820 Richmond Road, SW, Calgary, Alberta, Canada, T2T 5C7

$\dagger$ Microbiologist-in-Chief, Mt. Sinai and Princess Margaret Hospitals, Toronto, Ontario, Canada
}

potential for widespread dissemination of highly resistant organisms for which there is no adequate therapy is very real (6). To illustrate the epidemiology, risk factors and clinical management of antimicrobial resistant infections, this review will focus on resistance in the important community pathogen Streptococcus pneumoniae (pneumococcus).

\section{STREPTOCOCCUS PNEUMONIAE INFECTIONS}

There are more than one million annual deaths worldwide caused by $S$. pneumoniae in children less than five years of age (7). The majority of these deaths occur in developing countries and are due to pneumonia. Disease caused by $S$. pneumoniae may result from local spread of bacteria from the initial site of adherence in the nasopharynx, e.g., otitis media, sinusitis or pneumonia (8). Invasive disease occurs when $S$. pneumoniae reaches the bloodstream, resulting in bacteremia with no focus or a focal infection in one or more sites throughout the body. In developed countries, $S$. pneumoniae is the most common bacterial pathogen causing otitis media, bacteremia, pneumonia and meningitis $(7,8)$.

\section{INCIDENCE OF S. PNEUMONIAE INFECTIONS}

Recent population-based surveys have described the incidence rate and mortality of invasive $S$. pneumoniae infections in children and adults (9-15). Overall incidence rates (cases per 100,000 per year) differ between groups and regions: Australia (aboriginals), 222 (13); Australia (non-aboriginals), 16 (13); Alaska, USA (natives), 74 (14); Alaska, USA (non-natives), 16 (14); Toronto, Canada, 16 (15); and Southern California, USA, 13 (12). The incidence rate is highest in children less than two years of age and in the elderly, 
Table 1. Association of $S$. pneumoniae susceptibility with resistance (intermediate and high) to other antimicrobials according to the minimum inhibitory concentration (MIC) of penicillin. ${ }^{a}$

Percent of $S$. pneumoniae isolates resistant to other antimicrobials

\begin{tabular}{lccc}
$\begin{array}{l}\text { Other } \\
\text { Antimicrobials }\end{array}$ & $\begin{array}{c}\text { Penicillin susceptible } \\
(\mathbf{M I C}<\mathbf{0 . 1} \boldsymbol{\mu g} / \mathbf{m L})\end{array}$ & $\begin{array}{c}\text { Intermediately penicillin resistant } \\
\text { (MIC 0.1 } \mathbf{~ 1 ~} \boldsymbol{\mu g} / \mathbf{m L})\end{array}$ & $\begin{array}{c}\text { Highly penicillin resistant } \\
(\mathbf{M I C}>\mathbf{2} \boldsymbol{\mu g} / \mathbf{m L})\end{array}$ \\
\hline TMP/SMX & 12 & 54 & 97 \\
Macrolides & 2 & 12 & 25 \\
Chloramphenicol & 2 & 21 & 25 \\
Tetracycline & $<1$ & 3 & 19 \\
Ofloxacin & $<1$ & 8 \\
\hline \hline
\end{tabular}

a Data from Simor et al. (26)

with a male to female ratio ranging from $1.1: 1$ to $1.6: 1$ $(11,12)$. The overall mortality rate (cases per 100,000 per year) is 18 in Australia (aboriginals) (13), 6 in Alaska (natives) (14) and 1 to 2 in all other populations (12-15). The high disease rates in Australian aboriginals and Alaskan natives, compared to other populations, are likely related to factors such as crowding, lack of clean water and more prevalent chronic illnesses $(13,14)$. The rate of invasive $S$. pneumoniae infections is increasing in some regions. For example, in Norway, nationwide surveillance found an increase (in cases per 100,000 per year) from 3.5 in 1982 to 16.1 in 1995 (16). Similar findings have been reported from Denmark and Sweden $(17,18)$. The reasons for this increase are unknown, although a temporal association with the reduction in nasopharyngeal carriage and disease caused by Haemophilus influenzae type b (as a result of the very successful vaccine) has lead to speculation that S. pneumoniae has filled the ecological niche left vacant in the nasopharynx (19).

ANTIMICROBIAL RESISTANT S. PNEUMONIAE

Fully penicillin sensitive isolates of $S$. pneumoniae are defined by a minimum inhibitory concentration (MIC) of $<0.1 \mu \mathrm{g} / \mathrm{mL}$ (20). Intermediate resistance to penicillin is defined by an MIC of $>0.1$ to $<1.0 \mu \mathrm{g} / \mathrm{mL}$ and high-level penicillin resistance is defined by an MIC $>2.0 \mu \mathrm{g} / \mathrm{mL}$. Intermediate and high-level resistance to other antimicrobials are also defined according to MICs (20). For the sake of clarity, and to distinguish the (possibly) more clinically relevant resistant strains, $S$. pneumoniae with reduced susceptibility to penicillin is referred to as PNSP (penicillin nonsusceptible pneumococcus, MIC $>0.125$ $\mu \mathrm{g} / \mathrm{mL}$ ) or PRP (penicillin resistant pneumococcus, MIC $>2.0 \mu \mathrm{g} / \mathrm{mL}$ ) (21). The term PSSP refers to penicillin-susceptible $S$. pneumoniae infections.

The effect of penicillin is to bind to one or more of six known penicillin binding proteins (PBPs) located in the S. pneumoniae cell membrane (1A, 1B, 2A, 2B, 2X and
3) (22). The normal function of these PBPs is to enzymatically promote crosslinking of bacterial cell wall precursors, an integral step in cell wall development. This action is inhibited when PBPs are bound to penicillin. The binding of penicillin to individual PBPs occurs with variable avidity. Bacterial killing as a subsequent result of penicillin binding takes place in two ways (23). First, penicillin induces the release of autolytic bacterial enzymes (primarily amidase) which lyses the cell wall. Second, killing occurs in an autolysis-independent manner which is not well understood.

Resistance to penicillin (and to other $\beta$-lactam antimicrobials) occurs when the target penicillin binding proteins become physically altered so that penicillin binds with reduced affinity. These alterations occur as a result of chromosomal mutation, whereby exogenous DNA is taken up by individual $S$. pneumoniae and incorporated into the bacterial chromosome in a process known as transformation $(22,24)$. The development of intermediate or high-level resistance to penicillin occurs when alterations to at least 3 penicillin binding proteins that normally have high avidity for penicillin has occurred (1A, 2X and 2B) (25). Resistance to cephalosporins occurs with alterations to just two penicillin binding proteins (1A and 2X) (25).

In addition to $\beta$-lactam antimicrobials, many other antimicrobials have been used to successfully treat S. pneumoniae infections. Nevertheless, S. pneumoniae resistance to carbapenems, cotrimoxazole, macrolides, clindamycin, tetracycline, chloramphenicol, fluoroquinolones and rifampin has been reported (22). Resistance to multiple antimicrobials is common (Table 1) $(21,26)$, although reasons for the association of resistance to multiple antimicrobials are not completely understood. S. pneumoniae resistant to two or more classes of antimicrobials are considered multi-drug resistant S. pneumoniae (MDRSP) (27). It would seem logical that the acquisition of drug resistance would impose a cost on the bacteria as the result of a reduction 
in fitness caused by alterations to vital structures such as the cell wall (6). However, data suggest that natural selection often eliminates the growth disadvantage of resistant bacteria (28). The only antimicrobials to which $S$. pneumoniae remain universally susceptible are vancomycin and teicoplanin, drugs that have been used less often so far to treat these infections (29). Resistance to other antimicrobials is mediated by transposons (a DNA element which carries genes) or chromosome alterations which also generally result in altered binding sites $(22,24)$.

\section{EPIDEMIOLOGY OF PENICILLIN RESISTANT S. PNEUMONIAE INFECTIONS}

Penicillin-resistant $S$. pneumoniae were first induced in laboratory strains in 1943 (at the time penicillin was first introduced) (30). In 1965, a report from a Boston laboratory found that 2 of 200 clinical isolates were resistant to penicillin (31). The first reported clinically significant infection caused by a penicillin resistant strain of $S$. pneumoniae was described in Australia in 1967 (32). Between 1974 and 1984, case series of S. pneumoniae infections or isolates with $>10 \%$ penicillin resistance were reported from Israel, New Guinea, Poland, South Africa, Spain, and from some states in the USA (33). In 1977, multiresistant isolates were first found in South Africa (34).

The emergence of antimicrobial resistant $S$. pneumoniae may occur rapidly within a region. This has been well documented in the United States, where the prevalence of PNSP increased from $5 \%$ by 1987 , to $8 \%$ by 1992 , and to $25 \%$ (7\% to $10 \%$ PRP) by 1995 $(21,27,35-38)$. In addition, $26 \%$ of isolates found in a 1993-94 survey were MDRSP (21).

Worldwide, there is considerable variation in the incidence of PNSP. Surveys from Korea (70\%), Hungary (59\%), South Africa (45\%) and Spain (44\%) have reported the highest prevalence of PNSP (39-42). Other countries with a high prevalence include Hong Kong (29\%), Japan (26\%), and Bulgaria (24\%) (43-45). In some cases, adjacent countries have very different rates of resistance. For example, the rate of PNSP in Hungary is very high (59\%), while in Austria and Italy, the rates are low $(7 \%$ and $6 \%$ respectively) $(40,46,47)$.

In 1979, two cases of PNSP disease were reported in Canada $(48,49)$. Until recently, surveys have found the rate of PNSP in different areas of Canada to be $3 \%$ or less (48,50-52). However, since 1994, increased resistance has been reported in different regions. A survey of clinical isolates from community and hospital laboratories in Toronto during 1993 and 1994 found that $7 \%$ of isolates were PNSP (53). A later survey of clinical isolates obtained from 39 hospital and community laboratories across Canada during 1994 and 1995 found that 12\% of isolates were PNSP, however $20 \%$ of invasive isolates were PNSP $(95 \% \mathrm{CI}=13 \%, 27 \%)$, compared with $11 \%$ of noninvasive isolates $(95 \% \mathrm{CI}=9 \%, 13 \%)(26)$. $\mathrm{A}$ survey of invasive isolates obtained from 10 pediatric hospitals across Canada from 1991 to 1994 found that just $3 \%$ of isolates were PNSP (54). However, in 1995, 16\% of nasopharyngeal isolates and $11 \%$ of invasive isolates from a survey of children in Toronto were PNSP (55). Thus, Canada appears to be experiencing a period of rapid increase in PNSP rates as occurred earlier in the United States and elsewhere (Figure 1).

\section{RISK FACTORS FOR PENICILLIN NON- SUSCEPTIBLE $S$. PNEUMONIAE INFECTIONS}

Numerous socioeconomic and demographic factors have been shown to increase the risk of developing invasive $S$. pneumoniae infections (whether or not PNSP) including young age, young siblings, attendance at child care centre or day home, history of frequent otitis media, upper respiratory infection, HIV infection, sickle cell anemia, other immunosuppresive states, frequent hospitalization, smoking, chronic lung disease and chronic heart disease (7,56-63). The currently available capsular polysaccharide vaccine is $56 \%$ to $81 \%$ effective to prevent invasive $S$. pneumoniae infections over the age of two years (but has no benefit under the age of two years) and breast feeding of infants may be protective $(60,64)$.

Other studies have evaluated factors associated with invasive disease or otitis media caused by PNSP $(27,42,65-76)$. The results of most of these studies were limited by their retrospective design (67-74), non-population-based samples $(42,66-76)$, or small number of PNSP cases $(67,68,72,74,75)$. Nava and colleagues' population-based, prospective survey of 374 invasive S. pneumoniae cases ( 88 with PNSP), which obtained clinical information from a patient interview, has provided the best data to date (65). Factors were compared between those with PNSP and those with PSSP, using univariate analysis and multiple logistic regression. With multivariate analysis, the following factors were found to be associated with PNSP: age less than 5 years (odds ratio $(\mathrm{OR})=5.3,95 \%$ confidence interval $(\mathrm{CI})=2.2-12.6)$; immunosuppression $(\mathrm{OR}=3.0$, $\mathrm{CI}=1.5-6.0)$; and use of $\beta$-lactam antimicrobials in the prior three months $(\mathrm{OR}=2.1, \mathrm{CI}=1.0-4.5)$. Two other factors did not achieve significance in the multivariate model, although they were significant with univariate analysis: nosocomial acquisition $(\mathrm{OR}=1.2, \mathrm{CI}=0.9$ 4.5); and hospitalization in the prior 6 months $(\mathrm{OR}=1.1$, $\mathrm{CI}=0.6-2.3$ ). The type of infection was also included in the multivariate model and PNSP infections were no more likely to be meningitis and/or septicemia than pneumonia $(\mathrm{OR}=1.0, \mathrm{CI}=0.5-1.9)$. 


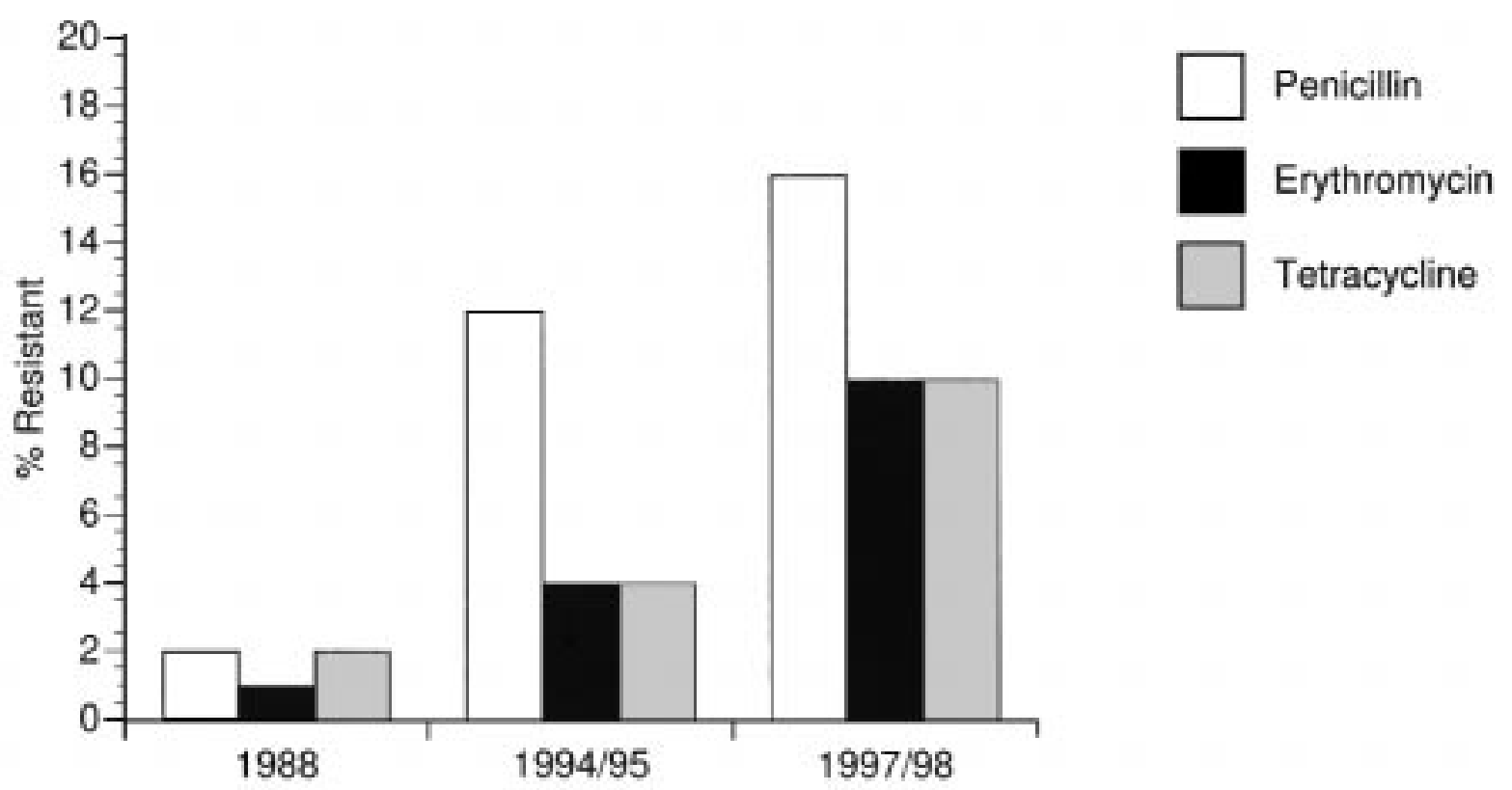

Figure 1. Trends in prevalence of S. pneumoniae resistance to penicillin, erythromycin and tetracycline in Canada (D. E. Low, unpublished data).

In addition to the above, the following risk factors have been associated with PNSP infections in at least two studies: nosocomial acquisition, recent hospitalization, pneumonia in prior year, child care centre attendance and caucasian race (27,42,66-76). Rather than racial differences in susceptibility, it is likely that higher socioeconomic status and access to medical care is the direct factor associated with PNSP infection. Access to medical care implies access to antibiotic therapy that those without access to medical care will not have. There is some evidence for this. In addition to caucasian race as a factor for PNSP, Hofmann found that those living in suburbs of Atlanta had PNSP infections more often than those living in urban Atlanta, regardless of race (27). The socioeconomic status of people residing in suburbs of Atlanta is higher than in urban areas. Mannheimer found that patients with PNSP infections were more likely to be caucasian than hispanic or black in a New York City hospital $(\mathrm{OR}=5.5$, $95 \% \mathrm{CI}=1.2,26.7)$ (74). Mannheimer also performed a survey of nasopharyngeal carriage of $S$. pneumoniae in children in a chronic care facility and found no racial differences in the carriage of PNSP in this setting (74). All children in the facility had equal access to medical care, including antimicrobials.

\section{OUTCOME OF PENICILLIN NONSUSCEPTIBLE S. PNEUMONIAE INFECTIONS}

There is limited data about the mortality and morbidity of PNSP infections. Mortality does not appear to be increased $(41,77)$. Pallares reported a ten year prospective cohort study of 464 cases of S. pneumoniae pneumonia (392 with bacteremia) (77). Thirty-five percent of cases were PNSP and $15 \%$ were PRP. Most cases were treated with high-dose penicillin (two million units every four hours) or equivalent doses of ampicillin, ceftriaxone or cefotaxime. In patients treated with penicillin, the mortality was $25 \%$ (6/24) in those with PNSP infections and 19\% (24/126) in those with penicillin-susceptible $S$. pneumoniae infections $(p=0.51)$. However, the power of the study to rule out a difference between the groups was just $10 \%$. In addition, multiple logistic regression found that penicillin resistance was not a risk factor for mortality $(\mathrm{OR}=1.3, \mathrm{CI}=0.7-2.2)$. The authors concluded that high dose penicillin or third-generation cephalosporins were adequate treatment for $S$. pneumoniae infections with penicillin MICs of $>2.0 \mu \mathrm{g} / \mathrm{mL}$.

The impact of PNSP infections can also be measured by outcomes other than mortality. For example, the rate of complications, requirement for hospitalization and use of multiple antimicrobials are clinically relevant outcomes given that they may have a significant impact on health care resources. One study has evaluated the morbidity of PNSP infections. Gómez prospectively studied 71 adults with $S$. pneumoniae bacteremia in a Spanish hospital (23 isolates were PNSP, 33\%) (75). No difference was found in the rate of complications between those with PNSP infections and those with PSSP infections (17/23 vs. 31/48, respectively, $p=0.43$ ), however the power to detect a difference 
between the groups was just $12 \%$. Further, no details about the complications were reported.

Some studies have evaluated the impact of different antimicrobial selections on the outcome of PNSP infections. Tan reviewed 19 invasive PNSP cases in children, all caused by intermediate-resistant S. pneumoniae (78). No comparisons were made with penicillin-susceptible cases, however, Tan concluded that PNSP bacteremia can usually be treated with $\beta$-lactams and meningitis can usually be treated with third-generation cephalosporins. Friedland reviewed 207 cases of invasive $S$. pneumoniae disease in children (83 with PNSP (40\%)) (41). There was no difference in mortality between those with PNSP (22\%) and those with penicillin susceptible infections $(15 \%, p=0.2)$. Friedland later performed a survey of children with non-meningitis invasive $S$. pneumoniae infections (66). Thirty-five of 108 (32\%) children had PNSP infections. In 75 children (25 PNSP), there were three categories of antimicrobial treatment evaluated (I-oral $\beta$-lactam; II-intravenous penicillin, ampicillin or cefuroxime; III-intravenous cefotaxime or ceftriaxone with or without vancomycin). There were no differences in the proportions improved, not improved or died after 3 and 7 days of therapy between children with PNSP and those with susceptible infections, regardless of the treatment regimen.

There have been numerous case reports of treatment failures using empiric third-generation cephalosporins (cefotaxime or ceftriaxone) for meningitis caused by S. pneumoniae with intermediate or high-level resistance to penicillin and cephalosporins (79). In all cases, clinical cure eventually occurred when vancomycin was added to the treatment regime.

Treatment failure with otitis media caused by PNSP appears to be more common when $\beta$-lactam antimicrobials are used $(80,81)$. Dagan reported a prospective study of 78 children with $S$. pneumoniae otitis media (80). All children had pre-treatment middle ear tympanocentesis which grew $S$. pneumoniae. There were 31 PNSP cases and 47 cases with susceptible $S$. pneumoniae. Follow-up clinical evaluations and repeat tympanocentesis were performed periodically after beginning treatment with cefuroxime axetil or cefaclor. Overall, bacteriologic failure (positive repeated culture) occurred in 14 cases, including 11 of 31 (35\%) PNSP cases and three of $47(6 \%)$ penicillin-susceptible cases $(p<0.05)$. Barry reviewed data from four clinical trials of treatment for otitis media in children (81). All children had pre-treatment middle ear tympanocentesis which grew S. pneumoniae. There were 54 PNSP cases and 182 cases with penicillin-susceptible $S$. pneumoniae. Each clinical trial had compared oral cephalosporins or amoxicillin-clavulinic acid. Treatment failure after 4 to
10 days was more common in the PNSP group than in the susceptible group (19\% vs. $8 \%$, respectively, $p=0.03$ ).

In summary, based on limited information, the mortality of PNSP infections does not appear to be increased. There is very limited information on other measures of outcome, but treatment failures do occur with PNSP infections, particularly meningitis and otitis media. These result in longer treatment courses with multiple antimicrobials. The influence of antimicrobial resistance on outcomes such as duration of antimicrobial treatment, requirement for multiple antimicrobials, duration of hospitalization, duration of acute symptoms and rate of complications is unknown.

\section{ISSUES IN MANAGEMENT OF ANTI- MICROBIAL RESISTANT S. PNEUMONIAE INFECTIONS}

Several consensus guidelines for the antimicrobial management of antimicrobial resistant S. pneumoniae have been recently published $(29,82-84)$. The remainder of this review will focus on the implications of resistance for treatment with common antimicrobials and general approaches to treatment of antimicrobial resistant $S$. pneumoniae.

\section{Meningitis}

Third-generation cephalosporins (cefotaxime or ceftriaxone) are standard choices for empiric treatment for bacterial meningitis after the neonatal period. However, as previously stated, there have been numerous case reports of treatment failures using third-generation cephalosporins for meningitis caused by $S$. pneumoniae with intermediate or high-level resistance to penicillin and cephalosporins which are related to inadequate bactericidal levels in the cerebrospinal fluid (79). PNSP isolates often remain susceptible to third-generation cephalosporins, but intermediate resistance may be common (21).

In regions where the prevalence of PNSP is increased, the empiric management of bacterial meningitis should include additional antibiotics combined with a thirdgeneration cephalosporin, or alternative antibiotics sufficient for monotherapy (84). Limited clinical data suggest that vancomycin or rifampin, added to cefotaxime or ceftriaxone achieve adequate bactericidal concentrations in the cerebrospinal fluid and may be additive or even synergistic (84-86). Combination therapy should be continued throughout the course of treatment if third-generation cephalosporin resistance is confirmed. Chloramphenicol may also be used in combination with a third-generation cephalosporin, however most labs are not able to determine susceptibility of $S$. pneumoniae to chloramphenicol for three to four days after an isolate is obtained (84). The 
carbapenems meropenem and imipenem are alternative choices for monotherapy of $S$. pneumoniae infections, including PNSP infections, although some PNSP strains are also resistant to carbapenems (84-87).

\section{Non-Meningeal Infections}

\section{$\beta$-lactam antibiotics}

The use of oral $\beta$-lactam antimicrobials for the treatment of PNSP non-meningeal invasive infections is controversial (84,88-90). Where clinical data are lacking, pharmacokinetics and pharmacodynamics may provide insight to the expected efficacy of a $\beta$-lactam (91-93). Animal studies with various penicillins and cephalosporins against PSSP and PNSP have found that the duration of time that serum antimicrobial levels are above the MIC is the key factor in determining efficacy. Mortality was virtually $100 \%$ if serum levels were above the MIC for $<20 \%$ of the dosing interval. In contrast, mortality was less than $10 \%$ when time above the MIC was $>40 \%$ (93).

It appears that non-meningeal infections caused by S. pneumoniae with intermediate resistance to penicillin can be treated with penicillin or amoxicillin. Apart from otitis media, discussed previously, there is little evidence for treatment failures, even with highly resistant strains. This is likely the result of the high drug concentrations achieved in the serum (91). However, to date, PRP strains with penicillin MICs $>4 \mu \mathrm{g} / \mathrm{mL}$ are exceedingly rare and the levels higher than $16 \mu \mathrm{g} / \mathrm{mL}$ have not been reported $(91,94)$.

For oral cephalosporins, there is a 4- to 16 -fold increase in the MICs for those S. pneumoniae strains that are intermediately resistant to penicillin, compared to their MICs against PSSP. There is another 2- to 8-fold increase in their MICs against strains that are highly penicillin resistant (95). Thus, oral cephalosporins should be used with caution, especially for the treatment of highly penicillin resistant strains. Amoxicillin is more active than any oral cephalosporin against PNSP (91). Craig determined the time above the MIC for several oral $\beta$-lactams (amoxicillin-clavulanate, cefaclor, cefuroxime, cefprozil, loracarbef, cefpodoxime, cefixime) and found that only amoxicillin/clavulanate was above the MIC for more than $40 \%$ of the dosing interval for PNSP $(93,96)$. Cefuroxime and cefprozil were above the MIC for $35 \%$ and $32 \%$, respectively, of the dosing interval, but only for intermediately resistant $S$. pneumoniae.

As the level of penicillin resistance increases (MIC $>8 \mu \mathrm{g} / \mathrm{mL}$ ), one of the ways to optimize the pharmacokinetic/pharmacodynamic parameters may be by increasing the antimicrobial dose and/or the dosing frequency. This has already been suggested when using amoxicillin for otitis media, and penicillin for
S. pneumoniae pneumonia $(89,97)$. Currently however, it would be appropriate to use any of the $\beta$-lactams for the treatment of PSSP pneumonia. For intermediately resistant strains, either amoxicillin or cefuroxime would be effective oral drugs. For highly resistant strains, either another class of compound, e.g., a macrolide or fluoroquinolone, for oral treatment, or either ampicillin, penicillin G, cefotaxime, or ceftriaxone for parenteral therapy, would be appropriate $(91,93)$.

At what penicillin MIC value and prevalence will it no longer be advisable to recommend a $\beta$-lactam for the empiric therapy of suspected $S$. pneumoniae pneumonia? Because of the mechanism of penicillin resistance in S. pneumoniae, resistance is low-level and often only one dilution above the breakpoint. As remodeling of the PBPs occurs, the MIC increases in modest increments from sensitive to intermediate to resistant. The amount of remodeling, and therefore the increase in the level of resistance that can occur is limited, since the function of the PBP must be maintained in order for the organism to survive. By increasing the dose of the $\beta$-lactam, effective therapy can still be achieved for infections such as bacteremia and pneumonia, where it is still possible to increase serum and tissue concentrations 4 to 8 -fold dilutions above the MIC. Currently, there is only anecdotal evidence that as the MICs rise to $>4 \mu \mathrm{g} / \mathrm{mL}$, the outcome of the treatment of such patients is jeopardized $(77,89)$. Currently, such levels of resistance do not exist and until levels of resistance are appearing that are associated with clinical failures, the currently recommended $\beta$-lactams for the treatment of penicillinresistant strains should not be abandoned.

\section{Macrolides}

The macrolide, lincosamide, and streptogramin A and B (MLS) antibiotics are three chemically distinct, functionally related drug classes which inhibit protein synthesis through alteration of the 50S ribosomal subunit (98). Resistance to one of the members of the class always includes other members of that class and sometimes other classes. The macrolides include erythromycin, clarithromycin, azithromycin, dirithromycin, and roxythromycin. The lincosamides include clindamycin. Resistance to these antibiotics occurs by target site modification, enzymatic inactivation, and active efflux (for a discussion of drug efflux, please see Van Veen et al. in this issue of the MJM) (99-102). When macrolide resistance is due to target modification there is complete cross-resistance between the macrolides and clindamycin. When the resistance is due to the presence of an efflux mechanism there is no cross-resistance with clindamycin, but cross-resistance to the streptogramins may occur. Resistance due to inactivation is class specific. 
Currently, monotherapy with a macrolide is the most common regimen used for the outpatient treatment of community acquired pneumonia after early childhood, including young ( $<60$ years of age) patients with and without comorbidity and in older patients (103). Since the choice of initial therapy is made without the benefit of knowing the pathogen and in view of the increasing prevalence of macrolide resistant $S$. pneumoniae, at what level of resistance will physicians no longer be able to use macrolides as empiric therapy? There is currently no answer to this question since it is not known if the macrolide resistant $S$. pneumoniae (evaluated in vitro) from a patient with pneumonia is associated with failure of therapy (104).

Why not just increase the dose or the frequency of dosing, as has been suggested for the penicillins? Macrolide susceptibility in $S$. pneumoniae is defined as a MIC $<0.5 \mu \mathrm{g} / \mathrm{mL}$. The mean MICs for macrolideresistant strains is 10 to $64 \mu \mathrm{g} / \mathrm{mL}$ (105). The very high levels of resistance (MIC $64 \mu \mathrm{g} / \mathrm{mL}$ ) occur in around $45 \%$ of resistant isolates and are due to target site modification resulting in dramatically reduced avidity of the macrolide for the target (106). Peak levels in serum of 2 to $3,0.5$ to 1 , and $0.4 \mu \mathrm{g} / \mathrm{mL}$ are reached at 3 hours after an oral dose of erythromycin $(500 \mathrm{mg})$, clarithromycin $(250 \mathrm{mg})$, and azithromycin $(500 \mathrm{mg})$, respectively (107). Therefore macrolide resistance results in a several fold increase in the MIC, well above achievable concentrations in the serum. Despite this and the number of macrolide-resistant $S$. pneumoniae worldwide and the frequency of $S$. pneumoniae pneumonia, reports of treatment failures are rare (104). A possible explanation is that the concentrations in the lung alveolar cells are several times higher then the serum concentrations and MICs of macrolide resistance strains, suggesting that in vitro susceptibility testing to determine MICs based on serum levels does not accurately predict in vivo activity in other tissues (108). On the other hand, in view of the low mortality $(<1 \%)$ of patients with community acquired pneumonia treated as outpatients, the failure to identify a pathogen in greater than $50 \%$ of cases in the community, the current low rates of macrolide resistance $(<11 \%)$, a worse outcome in this group of patients, other than mortality, may not be detected unless well designed outcome studies are performed $(21,109)$.

\section{Fluoroquinolones}

Resistance to fluoroquinolones in gram positive bacteria is a result of target modification and/or active efflux of the antimicrobial $(110,111)$. The target for the fluoroquinolones are the topoisomerases. Topoisomerases are enzymes in bacteria that are responsible for DNA coiling. There are four topoisomerases, two of which are targets for the fluoroquinolones, topoisomerase II (DNA gyrase) and topoisomerase IV (topo IV). DNA gyrase consists of two subunits, A and B, which are encoded for by the gyrA and gyrB genes. Topo IV consists of two subunits, ParC and ParE, which are encoded for by the parC and parE genes. In $S$. pneumoniae, resistance appears to be mainly due to mutational alterations of the gyrA and parC genes (112). Resistance mediated by target modification will increase the MIC of ciprofloxacin from $1 \mu \mathrm{g} / \mathrm{mL}$ (susceptible) to 4 to $128 \mu \mathrm{g} / \mathrm{mL}(112,113)$. Another mechanism of $S$. pneumoniae fluoroquinolone resistance involves the efflux of fluoroquinolones from the cell, which generally results in a lower level of resistance than topoisomerase mutations, increasing the MIC twofold to fourfold (110).

The clinical interest in the new gram-positive fluoroquinolones (levofloxacin, sparfloxacin, gatifloxacin, grepafloxacin, trovafloxacin, clinafloxacin, moxifloxacin) is whether there is increased activity against $S$. pneumoniae compared to earlier fluoroquinolones such as ciprofloxacin. These compounds are very potent against $S$. pneumoniae (MIC at which $90 \%$ of organisms are inhibited (MIC90) $<0.5$ $\mu \mathrm{g} / \mathrm{L})$, including isolates that are resistant to penicillin and macrolides (114). Initial trials support their efficacy for the treatment of community acquired pneumonia, including those due to $S$. pneumoniae (115-118). At present however, fluoroquinolones are not recommended for routine treatment of infections in children because of concerns with side effects, particularly arthropathy involving the hips (119). The incidence of this problem in humans appears to be exceedingly low and so fluoroquinolones may become more common for children in the near future.

\section{CONCLUSIONS}

Antimicrobial resistance is a rapidly evolving issue in clinical medicine. The appropriate response to the problem of antimicrobial resistance must be societywide. Research on the development, spread and persistence of resistance as well as on the development and testing of new antimicrobial agents is needed (4). Individual physicians can take several steps to control resistance including thorough handwashing between patient visits, prescription of narrow spectrum antibiotics when possible, isolation of hospitalized patients with multidrug resistant infections to prevent transmission, familiarization with local antibiotic resistance data and refusing patients' demands for unnecessary antibiotics (120). The general public can also take individual actions to control resistance including not demanding antibiotics, taking antibiotics exactly as prescribed and proper handling and preparation of food (120). Antimicrobial resistant $S$. 
pneumoniae is an example of a particularly important problem, because of the worldwide prevalence of this organism and the breadth of invasive and noninvasive diseases it causes. The approach to treatment of these infections requires consideration of the site of infection, knowledge of local resistance rates and deciding on treatment with high dose $\beta$-lactam antibiotics, alternative drug classes or combination antibiotic therapy.

\section{REFERENCES}

1. Report of the Director-General. The World Health Report: Life in the 21st Century - A Vision for All. Geneva: World Health Organization; 1998.

2. Pinner RW, Teutsch SM, Simonsen L, et al. Trends in infectious diseases mortality in the United States. JAMA 275: 189-193; 1996.

3. Cohen ML. Epidemiology of drug resistance: implications for a post-antimicrobial era (and others). Science 257: 1050-1082; 1992.

4. Bax RP, Anderson R, Crew J, et al. Antibiotic resistance - what can we do? Nature Medicine 4: 545-546; 1998.

5. Tablan OC, Chorba TL, Schidlow DV, et al. Pseudomonas cepacia colonization in patients with cystic fibrosis: risk factors and clinical outcome. Journal of Pediatrics 107: 382-387; 1985.

6. Morris A, Kellner JD, Low DE. The superbugs: evolution, dissemination and fitness. Current Opinion in Infectious Diseases 12 , in press.

7. Obaro SK, Monteil MA, Henderson DC. The pneumococcal problem. British Medical Journal 312: 1521-1525; 1996.

8. Peter G, Klein JO. Streptococcus pneumoniae. In: Long SS, Pickering LK, Prober CG, eds. Principles and Practice of Pediatric Infectious Diseases. New York: Churchill Livingstone; 1997: 828-835.

9. Voss L, Lennon D, Okesene-Gafa K, et al. Invasive pneumococcal disease in a pediatric population, Auckland, New Zealand. Pediatric Infectious Disease Journal 13: 873-878; 1994.

10. Eskola J, Takala AK, Kola E, et al. Epidemiology of invasive pneumococcal infections in Finland. JAMA 268: 3323-3327; 1992.

11. Dagan R, Engelhard D, Piccard E, et al. Epidemiology of invasive childhood pneumococcal infections in Israel. JAMA 268: 3328-3332; 1992.

12. Zangwill KM, Vadheim CM, Vannier AM, et al. Epidemiology of invasive pneumococcal disease in Southern California: implications for the design and conduct of a pneumococcal conjugate vaccine efficacy trial. Journal of Infectious Diseases 174: 752-759, 1996.

13. Torzillo PJ, Hanna JN, Morey F, et al. Invasive pneumococcal disease in central Australia. Medical Journal of Australia 162: 182-186; 1995.

14. Davidson M, Parkinson AJ, Bulkow LR, et al. The Epidemiology of invasive pneumococcal disease in Alaska, 1986-1990 - ethnic differences and opportunities for prevention. Journal of Infectious Diseases 170: 368-376; 1994.

15. McGeer A, Landry L, Goldenberg E, et al. Population-based surveillance for invasive pneumococcal infections in Toronto, Canada: implications for prevention. 36th Interscience Conference on Antimicrobial Agents and Chemotherapy. New Orleans: American Society for Microbiology, Abstract K009; 1996.
16. Iverson BG. Epidemiology of invasive bacterial infections caused by Streptococcus pneumoniae, Haemophilus influenzae, Neisseria meningitidis and Streptococcus pyogenes in Norway 1982-1995, and the influence of Haemophilus influenzae type b conjugate vaccine on their occurrence: analysis of national surveillance data. London: London School of Hygiene and Tropical Medicine; 1996.

17. Nielsen SV, Henrichsen J. Incidence of invasive pneumococcal disease and distribution of capsular types of pneumococci in Denmark, 1989-94. Edpidemiology and Infection 117: 411-416; 1996.

18. Hedlund J, Svenson SB, Kalin M, et al. Incidence, capsular types, and antibiotic susceptibility of invasive Streptococcus pneumoniae in Sweden. Clinical Infectious Diseases 21: 948953; 1995.

19. Baer M, Vuento R, Vesikari T. Increase in bacteraemic pneumococcal infections in children [Letter]. Lancet 345: 661; 1995.

20. National Committee for Clinical Laboratory Standards. Performance standards for antimicrobial susceptibility testing. Villanova, Pennsylvania: National Committee for Clinical Laboratory Standards, 1997.

21. Butler JC, Hofmann J, Cetron MS, et al. The continued emergence of drug-resistant Streptococcus pneumoniae in the United States: an update from the Centers for Disease Control and Prevention's Pneumococcal Sentinal Surveillance System. Journal of Infectious Diseases 174: 986-993; 1996.

22. Klugman KP. Pneumococcal resistance to antibiotics. Clinical Microbiology Reviews 3: 171-196; 1990.

23. Moreillon P, Markiewicz Z, Nachman S, et al. Two bacterial targets for penicillin in pneumococci: autolysis-dependent and autolysis-independent killing mechanisms. Antimicrobial Agents and Chemotherapy 34: 33-39; 1990.

24. Neu HC. The crisis in antibiotic resistance. Science 257: 10641073; 1992.

25. McDougal LK, Rasheed JK, Biddle JW, et al. Identification of multiple clones of extended-spectrum cephalosporin-resistant Streptococcus pneumoniae isolates in the United States. Antimicrobial Agents and Chemotherapy 39: 2282-2288; 1995.

26. Simor AE, Louie M, Low DE. The Canadian Bacterial Surveillance Network. Canadian national survey of prevalence of antibiotic resistance among clinical isolates of Streptococcus pneumoniae. Antimicrobial Agents and Chemotherapy 40: 2190-2193; 1996.

27. Hofmann J, Cetron MS, Farley MM, et al. The prevalence of drug-resistant Streptococcus pneumoniae in Atlanta. New England Journal of Medicine 333: 481-486; 1995.

28. Spratt BG. Antibiotic resistance: counting the cost. Current Biology 6: 1219-1221; 1996.

29. Friedland IR, McCracken Jr. GH. Management of infections caused by antibiotic-resistant Streptococcus pneumoniae. New England Journal of Medicine 331: 377-382; 1994.

30. Eriksen KR. Studies on induced resistance to penicillin in a pneumococcus type I. Acta Pathologica et Microbiologica 22: 398-405; 1945.

31. Kislak JW, Razavi LMB, Daly AK, et al. Susceptibility of pneumococci to nine antibiotics. American Journal of Medical Science 54: 261-268; 1965.

32. Hansman D, Bullen MM. A resistant pneumococcus. Lancet 2: 264-265; 1967.

33. Appelbaum PC. World-wide development of antibiotic resistance in pneumococci. European Journal of Clinical Microbiology 6: 367-377; 1987.

34. Jacobs MR, Koornhof HJ, Robins-Browne RM, et al. Emergence of mulitply resistant pneumococci. New England 
Journal of Medicine 299: 735-740; 1978.

35. Doern GV, Brueggemann A, Holley HP, et al. Antimicrobial resistance of Streptococcus pneumoniae recovered from outpatients in the United States during the winter months of 1994 to 1995: results of a 30-center national surveillance study. Antimicrobial Agents and Chemotherapy 40: 1208-1213; 1996.

36. Spika JS, Facklam RR, Plikaytis BD, et al. Pneumococcus Surveillance Working Group. Antimicrobial resistance of Streptococcus pneumoniae in the United States, 1979-1987. Journal of Infectious Diseases 163: 1273-1278; 1991.

37. Breiman RF, Butler JC, Tenover FC, et al. Emergence of drugresistant pneumococcal infections in the United States. JAMA 271: 1831-1835; 1994.

38. Centers for Disease Control and Prevention. Drug-resistant Streptococcus pneumoniae - Kentucky and Tennessee, 1993. Mortality and Morbidity Weekly Report 43: 23-25; 1994.

39. Lee HJ, Park JY, Jang SH, et al. High incidence of resistance to multiple antimicrobials in clinical isolates of Streptococcus pneumoniae from a university hospital in Korea. Clinical Infectious Diseases 20: 826-835; 1995.

40. Marton A. Pneumococcal antimicrobial resistance: the problem in Hungary. Clinical Infectious Diseases 15: 106-111; 1992.

41. Friedland IR, Klugman KP. Antibiotic-resistant pneumococcal disease in South African children. American Journal of Diseases of Children 146: 920-923; 1992.

42. Garcia-Leoni ME, Cercenado E, Rodeño P, et al. Susceptibility of Streptococcus pneumoniae to penicillin: a prospective microbiological and clinical study. Clinical Infectious Diseases 14: 427-435; 1992.

43. Kam KM, Luey KY, Fung SM, et al. Emergence of multipleantibiotic-resistant Streptococcus pneumoniae in Hong Kong. Antimicrobial Agents and Chemotherapy 39: 2667-2670; 1995.

44. Yoshida R, Kaku M, Kohno S, et al. Trends in antimicrobial resistance of Streptococcus pneumoniae in Japan. Antimicrobial Agents and Chemotherapy 39: 1196-1198; 1995.

45. Setchanova L. Clinical isolates and nasopharyngeal carriage of antibiotic-resistant Streptococcus pneumoniae in hospital for infectious diseases, Sofia, Bulgaria, 1991-1993. Antimicrobial Drug Resistance 1: 7984; 1995.

46. Marchese A, Debbia EA, Arvigo A, et al. Susceptibility of Streptococcus pneumoniae strains isolated in Italy to penicillin and ten other antibiotics. Journal of Antimicrobial Chemotherapy 36: 833-837; 1995.

47. Mittermayer $\mathrm{H}$, Jebelean $\mathrm{C}$, Binder $\mathrm{L}$, et al. Antibiotic susceptibility of pneumococci isolated in Austria over a fouryear period. European Journal of Clinical Microbiology and Infectious Diseases 15: 817-820, 1996.

48. Ahronheim GA, Reich B, Marks MI. Penicillin-insensitive pneumococci. American Journal of Diseases of Children 133: 187-191; 1979.

49. Pabst HF, Nigrin J. Penicillin resistance of pneumococci and immune deficiency. Lancet 2: 359-360; 1979.

50. Dixon JMS, Lipinski AE, Graham MEP. Detection and prevalence of pneumococci with increased resistance to penicillin. Canadian Medical Association Journal 117: 1159$1161 ; 1977$.

51. Jetté LP, Lamothe F, and The Pneumococcus Study Group. Surveillance of invasive Streptococcus pneumoniae infection in Quebec, Canada from 1984 to 1986: serotype distribution, antimicrobial susceptibility and clinical characteristics. Journal of Clinical Microbiology 27: 1-5; 1989.

52. Scheifele D, Gold R, Talbot J, et al. A national survey of invasive pneumococcal infections in children. Infectious Diseases Society of America Annual Meeting, San Francisco Abstract 410; 1995.
53. Simor AE, Rachlis A, Louie L, et al. Emergence of penicillinresistant Streptococcus pneumoniae in southern Ontario, 199394. Canadian Journal of Infectious Diseases 6: 157-160; 1995.

54. Scheifele D, Gold R, Marchessault V, et al. Penicillin resistance among invasive pneumococcal isolates at 10 children's hospitals, 1991-1994. Canada Communicable Disease Report 22; 1996.

55. Kellner JD, McGeer A, Cetron MS, et al. The use of Streptococcus pneumoniae nasopharyngeal isolates from healthy children to predict features of invasive disease. Pediatric Infectious Disease Journal 17: 279-286; 1998.

56. Takala AK, Jero J, Kela E, et al. Risk factors for primary invasive pneumococcal disease among children in Finland. JAMA 273: 859-864; 1995.

57. Andiman WA, Mezeger J, Shapiro E. Invasive bacterial infections in children born to women infected with human immunodeficiency virus type 1 . Journal of Pediatrics 124: 846852; 1994.

58. Black S, Shinefield H, Elvin L, et al. Pneumococcal epidemiology in childhood in a large HMO population. 34th Interconference on Antimicrobial Agents and Chemotherapy. Orlando: American Society for Micrbiology Abstract G71; 1994.

59. Farley JJ, King JC Jr, Nair P, et al. Invasive pneumococcal disease among infected and uninfected children of mothers with human immunodeficiency virus infection. Journal of Pediatrics 124: 853-858; 1994.

60. Gessner BD, Ussery XT, Parkinson AJ, et al. Risk factors for invasive disease caused by Streptococcus pneumoniae among Alaska native children younger than two years of age. Pediatric Infectious Disease Journal 14: 123-128; 1995.

61. Fekety Jr JR, Caldwell J, Gump D, et al. Bacteria, viruses, and mycoplasmas in acute pneumonia in adults. American Review of Respiratory Disease 104: 499-507; 1971.

62. Lipsky BA, Boyko EJ, Inui TS, et al. Risk factors for acquiring pneumococcal infections. Archives of Internal Medicine 146: $2179-2185 ; 1986$

63. Zarkowsky HS, Gallagher D, Gill FM, et al. Bacteremia in sickle hemoglobinopathies. Journal of Pediatrics 109: 579-585; 1986.

64. Centers for Disease Control and Prevention. Prevention of pneumococcal disease: recommendations of the Advisory Committtee on Immunization Practices (ACIP). Morbidity and Mortality Weekly Report 46: 1-25; 1997.

65. Nava JM, Bella F, Garau J, et al. Predictive factors for invasive disease due to penicillin-resistant Streptococcus pneumoniae: a population-based study. Clinical Infectious Diseases 19: 884-90; 1994.

66. Friedland IR. Comparison of the response to antimicrobial therapy of penicillin-resistant and penicillin-susceptible pneumococcal disease. Pediatric Infectious Disease Journal 14: 885-890; 1995.

67. Jackson MA, Shelton S, Nelson JD, et al. Relatively penicillinresistant pneumococcal infections in pediatric patients. Pediatric Infectious Disease Journal 3: 129-132; 1984.

68. Reichler MR, Rakovsky J, Sobotová A, et al. Multiple antimicrobial resistance of pneumococci in children with otitis media, bacteremia, and meningitis in Slovakia. Journal of Infectious Diseases 171: 1491-1496; 1995.

69. Bédos JP, Chevret S, Chastang C, et al. Epidemiological features of and risk factors for infection by Streptococcus pneumoniae strains with reduced susceptibility to penicillin: findings of a French survey. Clinical Infectious Diseases 22: 63-72; 1996.

70. Welby PL, Keller DS, Cromien JL, et al. Resistance to penicillin and non-beta-lactam antibiotics of Streptococcus pneumoniae at 
a children's hospital. Pediatric Infectious Disease Journal 13: 281-287; 1994.

71. Block SL, Harrison CJ, Hedrick JA, et al. Penicillin-resistant Streptococcus pneumoniae in acute otitis media: risk factors, suscepibility patterns and antimicrobial management. Pediatric Infectious Disease Journal 14: 751-759; 1995.

72. Pallares R, Gudiol F, Liñares J, et al. Risk factors and response to antibiotic therapy in adults with bacteremic pneumonia caused by penicillin-resistant pneumococci. New England Journal of Medicine 317: 18-22; 1987.

73. Tan TQ, Mason EO, Kaplan SH. Penicillin-resistant systemic pneumococcal infections in children: a retrospective casecontrol study. Pediatrics 92: 761-767; 1993.

74. Mannheimer SB, Riley LW, Roberts RB. Association of penicillin-resistant pneumococci with residence in a pediatric chronic care facility. Journal of Infectious Diseases 174: 513$519 ; 1996$.

75. Gómez J, Baños V, Gómez JR, et al. Clinical significance of pneumococcal bacteraemias in a general hospital: a prospective study 1989-1993. Journal of Antimicrobial Therapy 36: 1021$1030 ; 1995$

76. Kronenberger CB, Hoffman RE, Lezotte DC, et al. Invasive penicillin-resistant pneumococcal infections: a prevalence and historical cohort study. Emerging Infectious Diseases 2: 121124; 1996.

77. Pallares R, Liñares J, Vadillo M, et al. Resistance to penicillin and cephalosporin and mortality from severe pneumococcal pneumoniae in Barcelona, Spain (published erratum appears in New England Journal of Medicine 333: 1655; 1995). New England Journal of Medicine 333: 474-480; 1995.

78. Tan TQ, Mason EO, Kaplan SL. Systemic infections due to Streptococcus pneumoniae relatively resistant to penicillin in a children's hospital: clinical management and outcome. Pediatrics 90: 928-933, 1992.

79. John CC. Treatment failure with use of a third-generation cephalosporin for penicillin-resistant pneumococcal meningitis: case report and review. Clinical Infectious Diseases 18: 188193; 1994.

80. Dagan R, Abramson O, Leibovitz E, et al. Impaired bacteriologic response to oral cephalosporins in acute otitis media caused by pneumococci with intermediate resistance to penicillin. Pediatric Infectious Disease Journal 15: 980-985; 1996.

81. Barry B, Gehanno P, Blumen M, et al. Clinical outcome of acute otitis media caused by pneumococci with decreased susceptibility to penicillin. Scandinavian Journal of Infectious Diseases 26: 446-452; 1994.

82. Bradley JS, Kaplan SL, Klugman KP, et al. Consensus: management of infections in children caused by Streptococcus pneumoniae with decreased susceptibility to penicillin. Pediatric Infectious Disease Journal 14: 1037-1041; 1995.

83. Paris M, Ramilio O, McCracken GH. Management of meningitis caused by penicillin-resistant Streptococcus pneumoniae. Antimicrobial Agents and Chemotherapy 39: 2171-2175; 1995

84. American Academy of Pediatrics Committee on Infectious Diseases. Therapy for children with invasive pneumococcal infections. Pediatrics 99: 289-299; 1997.

85. Doit C, Barre J, Cohen R, et al. Bactericidal activity against intermediately cephalosporin-resistant Streptococcus pneumoniae in cerebrospinal fluid of children with bacterial meningitis treated with high dose of cefotaxime and vancomycin. Antimicrobial Agents and Chemotherapy 41: 2050-2052; 1997.

86. Klugman KP, Friedland I, Bradley J. Bactericidal activity against cephalosporin-resistant Streptococcus pneumoniae in cerebrospinal fluid of children with acute bacterial meningitis. Antimicrobial Agents and Chemotherapy 39: 1988-1992; 1995.

87. Pikis A, Akram S, Donkersloot JA, et al. Penicillin-resistant pneumococci from pediatric patients in the Washington, DC, area. Archives of Pediatric and Adolescent Medicine 149: 30$35 ; 1995$.

88. Goldstein FW, Garau J. 30 years of penicillin-resistant $S$. pneumoniae: myth or reality? Lancet 350: 233; 1997.

89. Klugman KP. Epidemiology, control and treatment of multiresistant pneumococci. Drugs 52 (Supplement 2): 42-46; 1996.

90. Strachan SA, Friedland IR. Therapy for penicillin-resistant Streptococcus pneumoniae. Journal of Medical Microbiology 43: 237-238; 1995

91. Goldstein FW. Choice of an oral beta-lactam antibiotic for infections due to penicillin-resistant Streptococcus pneumoniae. Scandinavian Journal of Infectious Diseases 29: 255-257; 1997.

92. Drusano GL, Craig WA. Relevance of pharmacokinetics and pharmacodynamics in the selection of antibiotics for respiratory tract infections. Journal of Chemotherapy 9 (Supplement 3): 3844; 1997

93. Craig WA. Antimicrobial resistance issues of the future. Diagnostic Microbiology and Infectious Disease 25: 213-217; 1996.

94. Coffey TJ, Dowson CG, Daniels M, et al. Horizontal transfer of multiple penicillin binding protein genes, and capsular biosynthetic genes in natural populations of Streptococcus pneumoniae. Molecular Microbiology 5: 2255-2260; 1991

95. Appelbaum PC. New prospects for antibacterial agents against multidrug-resistant pneumococci. Microbial Drug Resistance 1: 43-48; 1995.

96. Craig WA. Pharmacokinetics/pharmacodynamic parameters: rationale for antibacterial dosing of mice and men. Clinical Infectious Diseases 26: 1-12; 1998.

97. Bryan CS, Talwani R, Stinson MS. Penicillin dosing for pneumococcal pneumonia. Chest 112: 1657-1664; 1997.

98. Weisblum B. Erythromycin resistance by ribosome modification. Antimicrobial Agents and Chemotherapy 39: $577-$ $585 ; 1995$.

99. Courvalin P, Ounissi H, Arthur M. Multiplicity of macrolidelincosamide-streptogramin antibiotic resistance determinants. Journal of Antimicrobial Chemotherapy 16 (Supplement A): 91$100 ; 1985$.

100. Low DE. Quinipristin/dalfopristin: spectrum of activity, pharmacokinetics, and initial clinical experience. Microbial Drug Resistance 1: 223-224; 1995.

101. Sutcliffe J, Tait-Kamradt A, Wondrack L. Streptococcus pneumoniae and streptococcus pyogenes resistant to macrolides but sensitive to clindamycin: a common resistance pattern mediated by an efflux system. Antimicrobial Agents and chemotherapy 40: 1817-1824; 1996.

102. Sutcliffe J, Grebe T, Tait-Kamradt A, Wondrack L. Detection of erythromycin-resistant determinants by PCR. Antimicrobial Agents and Chemotherapy 40: 2562-2566; 1996.

103. Gleason PP, Kapoor WN, Stone RA, et al. Medical outcomes and antimicrobial costs with the use of the American Thoracic Society Guidelines for outpatients with community-acquired pneumonia. JAMA 278: 32-39; 1997.

104. Moreno S, Garcia-Leoni ME, Cercenado E, et al. Infections caused by erythromycin-resistant Streptococcus pneumoniae: incidence, risk factors, and response to therapy in a prospective study. Clinical Infectious Diseases 20: 1195-1200; 1995.

105. Johnston NJ, de Azavedo JC, Kellner JD, Low DE. Prevalence and characterization of the mechanisms of macrolide, lincosamide and streptogramin resistance in Streptococcus 
pneumoniae from across Canada. 37th Interscience Conference on Antimicrobial Agents and Chemotherapy Abstract. Toronto: American Society for Microbiology, 1997.

106. Johnston NJ, de Azavedo JC, Kellner JD, Low DE. Prevalence and characterization of the mechanisms of macrolide, lincosamide and streptogramin resistance in isolates of Streptococcus pneumoniae. Antimicrobial Agents and Chemotherapy, 42: 2415-2426; 1998.

107. Yao JDO, Moellering RCJ. Antibacterial Agents. In: Murray PR, Baron EJ, Pfaller MA, Tenover FC, Yolken RH, eds. Manual of Clinical Microbiology. Washington: American Society for Microbiology Press; 1995: 1281-1307.

108. Patel KB, Xuan D, Tessier PR, et al. Comparison of bronchopulmonary pharmacokinetics of clarithromycin and azithromycin. Antimicrobial Agents and Chemotherapy 40: 2375-2379; 1996.

109. Fine MJ, Auble TE, Yealy DM, et al. A prediction rule to identify low-risk patients with community-acquired pneumonia. New England Journal of Medicine 336: 243-250; 1997.

110. Zeller V, Janoir C, Kitzis MD, et al. Active efflux as a mechanism of resistance to ciprofloxacin in Streptococcus pneumoniae. Antimicrobial Agents and Chemotherapy 41: 1973-1978; 1997.

111. Pan XS, Ambler J, Mehtar S, et al. Involvement of topoisomerase IV and DNA gyrase as ciprofloxacin targets in Streptococcus pneumoniae. Antimicrobial Agents and Chemotherapy 40: 2321-2326; 1996.

112. Tankovic J, Perichon B, Duval J, et al. Contribution of mutations in gyrA and parC genes to fluoroquinolone resistance of mutants of Streptococcus pneumoniae obtained in vivo and in vitro. Antimicrobial Agents and Chemotherapy 40: 2505-2510; 1996.

113. Wise R, Brenwald N, Gill M, et al. Streptococcus pneumoniae resistance to fluoroquinolones [Letter]. Lancet 348: 1660; 1996.

114. Piddock LJ. New quinolones and gram-positive bacteria. Antimicrobial Agents and Chemotherapy 38: 163-169; 1994.

115. Adams M, Sullivan J, Henry D, et al. Comparison of grepafloxacin with cefaclor in the treatment of communityacquired pneumonia. 37th Interscience Conference on Antimicrobial Agents and Chemotherapy Abstract. Toronto: American Society for Microbiology Press; 1997.

116. Graham DR, Klein T, Torres A, et al. A double blind, randomized, multicenter study of nosocomial pneumonia (NOS) comparing trovafloxacin with ciprofloxacin \pm clindamycin/metronidazole. 37th Interscience Conference on Antimicrobial Agents and Chemotherapy. Toronto: American Society for Microbiology Press; 1997.

117. Mandell LA, Hopkins WD, Hopkins S. Efficacy of trovafloxacin in patients with community-acquired pneumonia due to penicillin-susceptible and resistant $S$. pneumoniae. 37th Interscience Conference on Antimicrobial Agents and Chemotherapy. Toronto: American Society for Microbiology Press; 1997.

118. File TM Jr, Segreti J, Dunbar L, et al. A multicenter, randomized study comparing the efficacy and safety of intravenous and/or oral levofloxacin versus ceftriaxone and/or cefuroxime axetil in treatment of adults with community-acquired pneumonia. Antimicrobial Agents and Chemotherapy 41: 1965-1972; 1997.

119. Schaad UB, Salam MA, Aujard Y, et al. Use of fluoroquinolones in pediatrics: consensus report of an International Society of Chemotherapy commission. Pediatric Infectious Disease Journal 14: 1-9; 1995.

120. Levy SB. The challenge of antibiotic resistance. Scientific American 278: 46-53; 1998.

James D. Kellner received his MD from the University of Calgary (Calgary, Alberta, Canada) and his M.Sc (Clinical Epidemiology) from the University of Toronto (Toronto, Ontario, Canada). He trained in pediatrics in Calgary and Toronto, where he was Chief Resident at the Hospital for Sick Children (Toronto, Ontario, Canada) . After a fellowship and practice in Emergency Pediatrics for five years, he received training in Infectious Diseases in Toronto. He is currently Assistant Professor in the Departments of Pediatrics and Microbiology and Infectious Diseases at the University of Calgary and a member of the Division of Infectious Diseases and the Child Health Research Unit at Alberta Children's Hospital (Calgary, Alberta, Canada). His research is in the fields of antibiotic resistance and the clinical and molecular epidemiology of Streptococcus pneumoniae infections. Donald E. Low is Microbiologist-in-Chief at Mt. Sinai and Princess Margaret Hospitals. He is also a professor in the Departments of Laboratory Medicine and Pathobiology at the University of Toronto. 